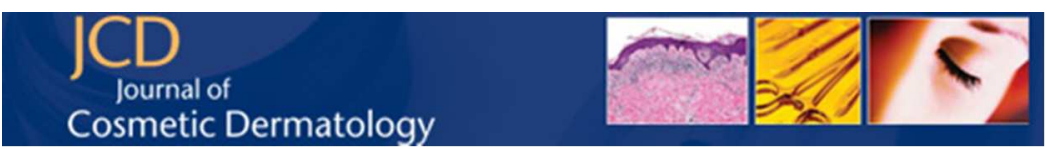

\title{
EXTERNAL LIPID FUNCTION IN ETHNIC HAIRS
}

\begin{tabular}{|r|l|}
\hline Journal: & Journal of Cosmetic Dermatology \\
\hline Manuscript ID & Draft \\
\hline Manuscript Type: & Original Contribution \\
\hline Keywords: & $\begin{array}{l}\text { ethnic hair, sebaceous lipids, hydrophobic character, mechanical } \\
\text { properties, water sorption/desorption, IR }\end{array}$ \\
\hline \multicolumn{2}{|l}{} \\
\hline
\end{tabular}

\section{SCHOLARONE ${ }^{\text {w }}$}

Manuscripts 


\section{INTRODUCTION}

Human hair is generally classified in three main, distinct ethnic groups: Caucasian African and Asian. Although several studies have been performed few studies have examined differences in hair morphology. These studies have concentrate primarily on, shape, section and dyameter, of the fiber, color and external stimuli responses, which help determine its mechanical properties ${ }^{1}$. Previous studies have largely centered on hair proteins, keratins and their molecular structures, such as amino acids ${ }^{2,3}$. Wolfram ${ }^{3}$ remarked on the uniformity in amino acid composition of hair of different ethnicities. Additionally, keratose fractions of solubilized hair were found to also be fairly similar between ethnicities. However, many differences have been found regarding the physical properties of the three types of fibers. Franbourg et al. ${ }^{2}$ found less radial swelling, for African hair, when flushed with water than Asian or Caucasian hair, and for this reason, the authors suggested that lipids differentiated among races. Syed et al. ${ }^{1}$ reported that moisturizing differences could possibly also be associated with differences in lipid structure or content.

Hair lipids across ethnic groups have been little studied ${ }^{4-6}$ because hair fibers contain low lipid contents and there are only few established methods that can accurately determine such lipid contents. Kreplak et al. ${ }^{6}$ examined lipid profiles in transverse cuts across hair using synchrotron infrared micro-spectrometry and determined that Caucasian hair often contains lipids localized inside the medulla and to a lesser extent, inside the cuticle. Furthermore, analyzed AfroAmerican hair does not exhibit this same hair lipid effect; the level of lipids is much lower, which contradicts earlier studies ${ }^{7}$. $\mathrm{Ji}$ et al. $^{4}$ presented analogous lipid composition in hair beyond groups. However, Asian hair was found to possess relatively more free fatty acids in the hair lipids which could be associated with their stronger protection to UV. Cruz et al. ${ }^{5}$ presented an important content of internal lipids in African hair.

Lipids are known to be part of the intercellular complex of the cuticle and the cortex. Fatty acids were reported to be linked to the proteins by thio-ester linkages at the external part of the cuticle $^{8}$. Considerable attention from the research community was given to these structural lipids, particularly covalently bound 18 -methyleicosanoic acid (18-MEA). Hair lipids primarily consist of cholesterol sulfate, ceramides, cholesterol, free fatty acids and cholesterol esters, which can be divided into endogeneous and exogeneous lipids according to their origin, hair matrix cells or sebaceous glands, respectively ${ }^{10}$. Their presence is directly linking with the 
internal water content inside the fiber. Internal hair lipids also enhance the barrier function to prevent foreign substances from permeating the fibers ${ }^{11,12}$

Lipids of sebaceous origin were often deemed as less important and thought to play little to no functional role in hair. In skin, sebum is an integral component that carries out a protective functional role $^{13}$. Sebaceous secretions provide a lipid-rich hydrophobic substance that protects the outermost surface of skin. Then a similar role could be expected for sebaceous lipids on hair. McMullen et al. ${ }^{14}$ supported a fundamental role of non-covalently bound lipids in the hair surface properties. These lipids not only regulate the hair properties but also influence hair care treatments. Most of the works on hair delipidization remove both sebaceous lipids and free structural lipids from the exterior and interior of the fibre ${ }^{14}$. For this reason, it is difficult to discriminate differences in the structural or functional or contributions of fiber properties by either free structural or sebum lipids.

Endogenous and exdogenous lipids from Caucasian human hair have been recently characterized studying their influence on hair properties ${ }^{15}$. External lipid depletion was found to induce a diminution of the total water content of the fibre. The water desorption increase was devoted to surface lipid disorder, due to a partial loss of external barrier. Besides, African hair was shown to contain the most total non-covalently bound lipids extracted from ethnic hairs, which was likely due from the external sebaceous lipids. Their extraction tends to ameliorate its fiber structure, which decreases its permeability to water and increases the tensile strength ${ }^{16}$. Then it could be interesting to determine differences in contribution of sebum lipids on ethnic hairs.

Therefore, the primary objective of this work is to study the contribution of exogeneous lipids from hair from different ethnic hairs on their physicochemical properties. In this study, the surface lipid content of different ethnic hairs (i.e., Asian, Caucasian and African) is characterized. Sebaceous lipids from the exterior of the fiber are removed. The influence of those free lipids on the hair properties, such as contact angle, mechanical characteristics and water sorption/desorption, will be evaluated to differentiate the permeation aspects of the fibers. In this work any changes in these physicochemical properties of delipidized hair by solvent extraction will be due to sebaceous free lipids, since covalently attached lipids (e.g., 18-MEA) will not be removed from the hair.

\section{MATERIALS AND METHODS}

\section{Materials}

The solvents used for this study were formic acid, diethyl ether, benzene, acetone, tert-butanol, methanol, and n-hexane (Merck, Darmstadt, Germany). The hairs used for this study are natural brown Caucasian hair, natural dark brown Asiatic hair, both without any previous treatment, and natural kinked Afro hair with only a thermic (without chemicals) pre-treatment. All of the hair samples were supplied by De Meo Brothers (Passaic, New Jersey, USA)

\section{Experimental}

The hair samples were washed in a solution of $3 \%$ diluted commercial shampoo Pantene Pro-V (Procter \& Gamble, USA) with $1 / 30$ bath ratio (hair/surfactant). The samples were then thoroughly rinsed 3 times with water and dried at room temperature (RT) The external surface lipids were removed from using soxhlet extraction with t-butanol and $n$-hexane for $4 \mathrm{~h}^{16}$. The different extracts were then combined in a chloroform-methanol mixture (2:1) before analysis.

The melting points of the dried extracts were measured in a Kofler (Riechert, Viene, Austria). The initial and final melting points of each lipid extract were determined, and the medium value was evaluated. Differential scanning calorimetry (DSC) was performed using a Mettler Toledo DSC, model DSC-281 ${ }^{\mathrm{e}}$ following the protocol described elswhere ${ }^{15}$. Besides, thermogravimetric analysis was performed with a TGA instrument (Model TGA/SDTA $851^{\mathrm{e}}$, Mettler Toledo, Spain) with the protocol previously described ${ }^{15}$.

Thin-Layer Chromatography coupled to an automated Flame Ionization Detector (TLC-FID) latroscan MK-5 analyzer (latron, Tokyo, Japan) ${ }^{16}$ were used for lipid analyses. The lipid extracts were spotted onto silica gel-coated Chromarods (Type S-III) using a precision Hamilton 2- $\mu \mathrm{I}$ syringe coupled to an SES 3202/IS-02 sample spotter (Nieder-OIm, Germany). Lipid content was obtained using an optimized TLC/FID protocol ${ }^{15,16}$. 
The moisture content was determined from hair samples (non-extracted and extracted) of different ethnicities. A hair sample $(0.5 \mathrm{~g})$ was maintained in a conditioned room $\left(23^{\circ} \mathrm{C}, 50 \%\right.$ Relative Humidity $(\mathrm{RH})$ ) for at least $24 \mathrm{~h}$ before being weighed and then dried in an oven at $105^{\circ} \mathrm{C}$ for $12 \mathrm{~h}$. The moisture content was calculated as a percentage after maintaining the sample in a desiccator under a $\mathrm{P}_{2} \mathrm{O}_{5}$ atmosphere and weighed again.

Contact angle was determined to obtain information regarding the surface energy of the solids. were calculated from The dynamic wetting force measurements performed in an electrobalance KSV Sigma 70 contact angle meter allows to obtain the contact angle values ${ }^{17}$. "Pseudo-static" contact angle formed by water droplets on parallel aligned and fixed hair collectives were also performed $^{18}$. The side of the droplets was recorded by a digital camera (Power Shot SX60, Canon, Spain).

Mechanical properties (breaking elongation and breaking stress) were evaluated using a dynamometer (Instron 5500R) in accordance with the ASTM Standard D 3822 (1980). Tensile testing were applied to thirty bundles of five parallel hair fibers previously conditioned for more than $48 \mathrm{~h}$ in a standard room $\left(20^{\circ} \mathrm{C}, 65 \% \mathrm{RH}\right)$ with $100 \mathrm{~mm}$ distance between the clamps. The bunch of fibres was strained at $60 \mathrm{~mm} / \mathrm{min}$. When broken, the hairs were cut, weighed and the mean hair linear density of the five fibers was obtained. Tex, (mean hair linear density $(\mathrm{mg} / \mathrm{m})$ ) and initial modulus (IM, the initial increase in tenacity per unit of strain $(\mathrm{mm} / 100 \mathrm{~mm})(\mathrm{cN} / \mathrm{tex}))$ were obtained. Besides, parameters related to the third (median) breakage were also reached: breaking load $(\mathrm{L})$, the load at which the median breakage occurs $(\mathrm{cN})$; breaking tenacity $(\mathrm{T})$, related to $L$ via the linear density of the three hairs in (cN/tex); and breaking deformation (D), the extension at which the median breakage occurs related to the initial sample length in \%.

A thermogravimetric balance, Sorption Analyzer Q5000SA (TA Instruments, New Castle, USA) with a controlled humidity chamber was applied to determined water desorption and absorption in the hair fibers. Experiments were conducted on weight of $10 \pm 1 \mathrm{mg}$ of hair samples with a total gas flow of $200 \mathrm{ml} / \mathrm{min}$, at $25^{\circ} \mathrm{C}$ following the protocol described elsewhere ${ }^{12,15}$.

To describe sorption isotherms, some mathematical models based on theoretical and/or empirical criteria have been published. One of the most commonly used is the Guggenheim, Anderson, de Boer model (GAB). Its parameters, with theoretical basis, provide a more physical aspect to the sorption process in comparison to other empirical models. The GAB model gives a special value to the monolayer moisture content of the fibre. Therefore, in this work, the sorption isotherm data were modeled following the GAB model ${ }^{19}$. Description of the GAB equation and the parameters used to fit the experimental sorption data are listed in Table I.

The method applied by Vickerstaff ${ }^{20}$ which studied the dye diffusion into the fibers has been adjusted in this work to obtain the diffusion coefficient of water expressed from a derived Fick's equation for moisture diffusion. Good results for the first stages of moisture absorption, were obtained with this expression. The amount of absorbed water plotted against the square root of the absorption time, should lie on a straight line in which the slope is the square root of the apparent diffusion coefficient, DA, measured in min-1over the sample's mass.

$$
R(t) / R_{f}=\sqrt{D_{A}} \sqrt{t}
$$

Synchrotron-Based Fourier Transform Infrared Microspectroscopy ( $\mu \mathrm{FTIR}$ ) was applied to all hair samples. Cryosecttion was performed with hair tufts of $1 \mathrm{~cm}$ which were embedded in Optimal Cutting Temperature compound, OCT (Bright Instruments, Bedfordshire, UK) and immediately frozen using liquid N2. Samples blocks were cut in $5 \mu \mathrm{m}$ cross sections using a Cryostat CM3050 S (Leica Biosystems Nussloch, Germany). Sections were placed on CaF2 circular windows, $1 \mathrm{~mm}$ thickness and $13 \mathrm{~mm}$ diameter (Crystran, Dorset, UK). SR- $\mu$ FTIR were performed at the MIRAS beamline at ALBA synchrotron (Catalonia, Spain), using a Hyperion 3000 Microscope equipped with a 36x magnification objective coupled to a Vertex 70 spectrometer (Bruker) with mercury-cadmium-telluride (MCT) detector that was continuously purged with nitrogen gas. Measuring range was $850-4000 \mathrm{~cm}^{-1}$ and the spectra collection was carried out in transmission mode at $4 \mathrm{~cm}^{-1}$ resolution, $10 \mu \mathrm{m} \times 10 \mu \mathrm{m}$ aperture dimensions and 128 scans. Background spectra were collected from a clean area of the CaF2 window every 20 scans. Spectra's were collected and classifieds using OPUS Spectroscopy software (Bruker). Baseline corrections were performed using OPUS and the spectra's of each map were separated according to the cuticle or cortex regions. Then, each spectra was analyzed using 
OMNIC software version 8.1.210 (Nicolet, Madison, WI). Base line of spectra's using the software correction tool and the peak characterization were performed using GaussianLorentzian fitting.

Once the results were obtained the Kruskal-Wallis test were applied to detect significant differences between samples. Statgraphics plus 5 software (Statpoint Technologies Inc. Warrenton, Virginia, USA) was used for statistical analyses. Significant differences in the mean values were evaluated by the $F$ test.

\section{RESULTS}

\section{Hair lipid analysis by TLC-FID}

Extraction of lipids from African, Asian and Caucasian hair was performed after washing with shampoo. The external surface lipids were removed from the ethnic hair surface using soxhlet extraction with t-butanol and $\mathrm{n}$-hexane as detailed in the experimental section. This methodology was performed in duplicate.

It is important to note the great difference obtained of the external lipids between the African and Asian/Caucasian hair samples (Table II), which is also reflected in the published data of total extracted lipids ${ }^{1621}$. It must be remarked that all fibers were previously washed with shampoo.

In order to correlate the differences in lipid composition of those external lipids between ethnicities, the lipid extracts were analyzed using TLC/FID in triplicate. This technique has also been recently used to analyze lipids from hair, ${ }^{5},{ }^{22}$. The external lipids, such as sterol esters and squalene (apolar lipids), are known to comprise most of the surface sebaceous lipids. The results (expressed in percentage of lipids in the total lipid extract) are detailed in Table II and in Figure 1 (expressed in percentage of lipids over the entire mass of the hair fiber).

The external lipids of the African hair fibers contain the greatest amount of apolar lipids with a consequently lesser amount of free fatty acids and polar lipids, whereas Caucasian and particularly Asian extracts contain a much greater amount of FFA and polar lipids, which is in accordance to Ji et al. ${ }^{4}$ (Table II).

Similar conclusions can be obtained from Figure 1. The differences in the external lipids related to the entire fiber weight are even more pronounced and significant for African hair when compared with Asian and Caucasian hair.

The large differences are noteworthy for the lipid analyses between the three types of ethnic hair fibers. Regarding the entire fiber, African hair contains the greatest proportion of apolar lipids (e.g., esterol esters and squalane) and free fatty acids and additionally, an even slightly greater amount of sterols and polar lipids.

This analysis was performed with the extract obtained from fibers that had been washed with shampoo. It is well known that African hair contains a greater amount of sebaceous lipids ${ }^{8}$, which accounts for the high measured amount of apolar lipids, squalene, cholesterol esters, etc. The large extraction of free fatty acids and apolar lipids from total lipid analyses of African hair was previously mentioned ${ }^{16}$ and it could be due to the external lipid fraction. This has been decidedly confirmed in the present work.

The melting point and enthalpies of the phase transitions of the extracts might be of importance when settling the physicochemical properties of the extracted lipids (Table III). The melting points of lipid mixtures are difficult to establish because each lipid melts at a different temperature; therefore, in Table III, the mean value (by triplicate) of the starting and ending melting temperatures of each extract is shown. The melting point is related to the unsaturation degree of the carbon chains of the different lipids, and these unsaturations are crucial on the barrier properties of the fibers (the higher the melting point, the lower the unsaturation degree and the lower the permeability could be expected). External lipids from Caucasian hair have a much higher melting point than that of African and Asian hair.

The DSC evaluation indicates that all extracts exhibit two phase transitions: a main peak $(-17$ to $\left.-32 \mathrm{Jg}^{-1}\right)$ at a low temperature $\left(10\right.$ to $\left.20^{\circ} \mathrm{C}\right)$ and a second smaller peak $\left(-6\right.$ to $\left.-12 \mathrm{Jg}^{-1}\right)$ at a slightly higher temperature $\left(30\right.$ to $\left.38^{\circ} \mathrm{C}\right)$. The primary difference is the extra phase transition for the Caucasian external lipids at $55^{\circ} \mathrm{C}$ and $-6 \mathrm{Jg}^{-1}$. The existence of this highest temperature 
peak for Caucasian external lipids and the highest enthalpy for the low-temperature peak of the Asian external lipids can be well related to the melting temperatures found for these extracts.

The first derivative of the TGA evaluation of all extracts also exhibits two primary degradation steps for all extracts, the more important step at 237 to $247^{\circ} \mathrm{C}$ and the second step from 330 to $360^{\circ} \mathrm{C}$. Moreover, as in the DSC case, only the Caucasian external lipids exhibited a peak at the higher temperature of $455^{\circ} \mathrm{C}$ even though it also accounts for $7.5 \%$. Again, these results are in agreement with the previously reported DSC and melting point results.

Degradation temperatures, enthalpies of phase transitions and the melting point of the lipid extracts are difficult to associate with the lipid amount extracted or with the chemical composition of the extracts, which have been described. This could be much related with the unsaturation degree of the compounds in a particular lipid family, which is likely the FFA.

Physicochemical properties of nontreated ethnic fibers and external lipid-extracted fibers were also determined. The moisture content $(\mathrm{H})$ for extracted and non-extracted hair fibers were evaluated (Table IV). It has to be mentioned that hair fibers moisture content ranges between 10 and $12 \%$ (measured at $50 \% \mathrm{RH}$ and $22{ }^{\circ} \mathrm{C}$ ). The Caucasian fibers were found to be the most hydrated fiber at approximately $11.7 \%$, which is different from Asian fibers and African fibers. Similar values for moisture content of ethnic hair were previously reported ${ }^{1,16}$.

When fibers are externally extracted, there is consistently a decrease in the moisture, which accounts for approximately $5 \%$ for Asian and African fibers and for more than $13 \%$ for Caucasian fibers; being this difference significant. This fact is difficult to relate with the different amounts of lipids extracted. Caucasian hair with a low amount of external extracted lipids shows that the higher moisture content decreases. A decrease in the water content could be greatly related to the lipid saturation degree rather than the total amount of lipids.

The contact angle was determined in an electrobalance by dynamic wetting force measurements and by the "pseudo-static" methodology, which evaluates images of water droplets on hair collectives that are aligned in parallel. Contact angle measurements indicated the marked hydrophobicity of all ethnic fibers, which is significantly superior for the African and Caucasian fibers compared with that of the Asian fibers. Delipidization promotes a slight decrease in this hydrophobicity, which reached a maximum decrease of $10 \%$ for the Caucasian fibers. It must be remarked that in addition to solvent-extractable lipids, there are additionally covalently-bound lipids at the surface, which form the f-layer. This arrangement, which was not modified, imparts are known to pronounce hydrophobicity to the hair surface, which is in marked contrast to the overall hydrophilicity of the bulk hair.

Hairs may be regarded as a combination of microfibrils ordered in parallel to the fiber axis imbedded in a matrix that includes lipids. Deformation and distortions to the fiber at the macroscopic level are transferred to the micrifibrils and the matrix. There are some primary parameters that enable characterization of the initial, yield and the post yield (breaking) regions.

Hair fineness determines the hair thickness which could vary after lipid extraction. The initial modulus evaluates the secondary bonds efficiency to support the distortions of the bonding network of the matrix. Deformation at break primarily is related to the size of the yield region when higher strains causes unfold of fibril chains. The hair behaves then like a plastic material, a liquid or an amorphous solid which is affected by the plasticizer effect of the matrix. Breaking tenacity shows the new hair configuration in the post-yield region.

To determine the consequence of ethnicity and lipid extraction on the hair mechanical properties, an analysis of variance was performed to the described parameters to relate the variations with the treatment and type of hair. The mean values and confidence intervals at $95 \%$ are shown in Table V.

African hair consists of coarse fibers with a lower initial modulus and lower deformation at break, which means a lower bonding level of the matrix and also a lower plasticizers effect in the matrix. Lipid extraction did not significantly affect the weaker African fibers even though much of the lipids were extracted. However, lipid extraction decreased the deformation at break of the Asian fibers and unexpectedly increased the break tenacity of the lipid-extracted Caucasian fibers. This increase in strength, for the extracted Caucasian fibers, was previously reported $^{16}$ and could be related to the also increment in abrasion resistance for another lipid extracted keratin fiber, such as wool. Following lipid removal an increment on intercellular adhesion was proposed to occur ${ }^{23}$. It should be highlighted the possible relationship between 
the decrease in humidity with the increase in break tenacity, especially important for the extracted Caucasian fibers.

Hair water sorption properties of hair can reflect damage due to different reactive cosmetic treatments ${ }^{3}$. Then, water desorption and absorption action of ethnic hairs with and without lipid extraction were evaluated to determine the humidity content of the fibers, the binding energies of water to the different fiber components and most importantly, the water diffusion, which is related with the integrity of the hair samples. Water absorption and the desorption for the three native and lipid-extracted ethnic hairs were acquired by the sorption analyzer. Moisture sorption hysteresis indicates the different behavior between absorption and desorption of the fiber. The three types of hair presented a shape of the water equilibrium water sorption isotherms described by a type II isotherm, with a low amount of water at low relative humidity and a large increase at a high relative humidity (Figure 2).

A clear maximum water regain for Caucasian fibers were found compared with the other two types of native fibers (Figure 2A, Table VI). This is in agreement with the higher humidity content found for Caucasian hairs in compared with that of the other two types of hairs (Table IV). A maximum water regain decrease was obtained for the external extracted hairs, mainly for African and Caucasian fibers (Table VI). These results are also in accordance with the humidity values obtained.

Values of monolayer capacity $(\mathrm{Wm})$, and the energy constants $(\mathrm{Cg}$ and $\mathrm{K}$ ) obtained by the regression of the experimental sorption data by the GAB model are listed in Table VI. External lipid extraction induces to a decrease in the water content of the monolayer, similarly to the maximum water regain. On the contrary, the energy constants, $\mathrm{K}$ and $\mathrm{Cg}$, of the primary and secondary sorbed monolayer increase after external lipid extraction. All of these results indicate an increase in the energy of binding water to the active groups of the extracted fibers, which results in more polar fibers.

Kinetic evaluation of the kinetics of the moisture absorption and desorption is a good way to obtain knowledge about the structural integrity of a given sample such as hair ${ }^{24}$. There are differences in the velocity at which African hair reaches equilibrium in front of the other two fiber types $\left(\mathrm{t}_{\mathrm{T}}\right)$. Asian and particularly Caucasian fibers more slowly reached equilibrium than the African fibers (Table VI). Moreover, evaluation of the diffusion coefficients confirmed differences in the keratinized structure of these three ethnic type of hairs. Typically, there is an inverse relationship between the diffusion coefficient and the time parameter; more time is needed to reach equilibrium for samples with low water permeability that means small diffusion coefficient. This happens in the ethnic hair fibers, where Caucasian and Asian hairs presented lower diffusion coefficients compared with those of African hair. Then, it can be said that African fibers have a higher permeability when compared with that of the other fibers.

Two diffusion coefficients were calculated $D_{A}$ which accounts for the apparent diffusion coefficient of the absorption and desorption process and $D_{A D}$ which only accounts for the desorption one which is more reliable for the fiber state. Comparison of diffusion coefficients of fibers with the lipid depleted ones indicate only small differences for the three fibers. This means that even the clear moisture diminution due to increase desorption, kinetics are not so clearly modified due to exogenous lipid depletion. Results obtained in previous works in which the total lipid content was depleted, indicated a more important diffusion decrease ${ }^{16,15}$. This means that sebaceous lipids do not have a main role in the fiber permeability. Diffusion of other keratinized tissues, such as nails ${ }^{24}$, wool ${ }^{12}$ and stratum corneum ${ }^{25}$, have been determined before and after being lipid extracted. In all cases, however in a different extent, the permeability tended to merge after lipid depletion, which revealed a partial structural loss.

Water has been known to enter into the hairs through the lipid bilayers from the fibers. The lipid bilayers from the stratum corneum of skin were described to contain non-fluid zones and fluid zones with differences in their permeability. The order in which the lipids are disposed can be determined by FTIR. The position of $\mathrm{CH}_{2}$ symmetric stretching mode $\left(\mathrm{CH}_{2}\right.$ St.Sym around 2850 $\mathrm{cm}^{-1}$ ) would indicate orthorhombic, or lamellar or gel lipid order with increasing permeability. Integration of $\mathrm{CH} 2$ asymetric stretching mode $\left(\mathrm{CH}_{2}\right.$ St.Asym around $\left.2920 \mathrm{~cm}^{-1}\right)$ is much reliable to determine lipid content. Water or polar compounds easily penetrates through the fluid zones. However, the non-fluid zones are think to be much related with the barrier properties of the keratin fibers. Synchrotron-Based Fourier Transform Infrared Microspectroscopy ( $\mu$ FTIR) were used to obtain the spectra's of each zone separated according to the cuticle or cortex regions. 
Results of the frequency of $\mathrm{CH}_{2}$ St.Sym around $2850 \mathrm{~cm}^{-1}$ of the cuticle and cortex regions of native and delipidized ethnic fibres are expressed in Table VII. Moreover, quantification was performed with the $\mathrm{CH}_{2}$ St.Asym integration of the peak normalized with the one of Amide I.

Native fibres present fewer amounts of lipids in the cortex related to cuticle of all fibres, presenting the African native fibres the highest quantity of lipids in the two zones when related with the other two fibres. African and Asian fibers have similar lipid order in cuticle and cortex. However, Caucasian present more order in the cortex than in the cuticle (lower frequency in the Cau cortex NT than in Cau cut NT). In summary, the presence of lipids is always more marked in the cuticle decreasing from African to Caucasian and to Asian fibres. This is in accordance with the amount of lipids extracted and analyzed previously. The lipid order is similar in the two zones of the three fibers being the maximum order in the lipid fraction of the Cortex of Caucasian fibres.

Delipidation of the fibres indicate a decrease in lipid content, in both regions of the three fibres being this much important for the African fibres followed by the Caucasian and with almost no difference for the Asian ones. It is important to remark that lipids were not only extracted from the cuticle, these results indicate that lipids are removed also in the cortex. Therefore, the soxhlet procedure extraction with t-butanol and $n$-hexane for $4 \mathrm{~h}$, believed to extracted external surface lipids also extracts internal lipids although mainly apolar in character.

The wavenumber of the delipidized fibres is very similar for African and Asian fibres. However, the Caucasian one presents a diminution in frequency in the cuticle leading to a more order orthorhombic package of Caucasian delipidized fibre. This fact can be related with the increase in breaking tenacity for delipidized Caucasian fibres, the bonding level of the microfibrils can be somehow increased due to an increase in the order of lipid arrangement.

The exogeneous lipids from different ethnic hairs were evaluated and also, the physicochemical properties of extracted fibers were determined. Structural and functional inputs of the obtained fiber properties by external lipids of ethnic hairs were discerned. Therefore, differences in the properties of ethnic hairs can be associated to their lipid structure.

\section{CONCLUSIONS}

In the present work, evaluation of native ethnic fibers was performed, which lead to the following pronounced results. Lipid extraction showed that African hair contained the highest amount of external lipids which makes the fiber more hydrophobic. Moreover, African hair has a lower initial modulus and lower deformation at break, which means it has a reduced strength. Moreover, African hair was shown to be the most permeable fiber due to the higher diffusion coefficients compared with that of the other fibers, even though IR results indicate that lipids from African hair are similarly packaged to the other fibres with orthorhombic rearrangement. Caucasian fibers were found to be the most hydrated fiber as shown by the water uptake and desorption results with less permeability. Optimum water permeability is essential in preventing the moisture content of the fiber from being altered due to rapid changes in the atmosphere.

Moreover, this work was based on investigating the influence of exogeneous lipids from different ethnic hairs to discern their contribution on physico-chemical properties. During the extraction step, sebaceous lipids of the fiber were removed and analyzed. IR results indicate that lipids are removed both in the cortex and in the cuticle. Therefore, the soxhlet procedure extraction with t-butanol and n-hexane, believed to extracted external surface lipids were found to also extract internal lipids although mainly apolar in character. Caucasian fibre was the only one that presents differences in lipid rearrangement after delipidization, a diminution in

It can be pointed out the great difference in the extracted amount of lipids between the African (3.8\%owf) and Asian (0.5\%owf)/Caucasian (0.9\%owf) hair samples. The sebaceous African extract contained the greatest fraction of free fatty acids, apolar lipids (e.g., squalene and esterol esters) and even a slightly greater amount of sterols. Results of lipid extracts melting points are not easily related with the chemical composition nor the amount of lipids extracted. Unsaturations in each lipid family could also affect the permeation properties differently. Caucasian external lipids exhibited by DSC and TGA peaks at the higher temperature than the other fibres. This is in accordance with the lowest diffusion properties from Caucasian fibres. 
Moreover, humidity retention decreases when the fibers are externally extracted by approximately $5 \%$ for African and Asian and by greater than $13 \%$ for Caucasian hair. The lipid extraction does not much affect the mechanical properties of the weaker African fiber despite the large amount of lipids that was extracted. However, lipid extraction decreases deformation at break of Asian fibers and unexpectedly, leads to an increase of the break tenacity of the extracted Caucasian hairs. This is in accordance with the increase in structural order after lipid depletion demonstrated by IR in cuticle of Caucasian fibres.

Hair delipidization is interesting not only for structural hair knowledge but also for representing the situation of many consumers after being submitted to harsh procedures or aging of the hair. Moreover, there is a lack of knowledge of ethnic hair structure, particularly African hair. The results of this research have particular relevance for chemists and new product development specialists who work in the ethnic hair care market. It is clear that the three types of hair, i.e., African, Asian and Caucasian hair, differ in some important properties, which suggests that the products, generally formulated for Caucasian hair, may not adequately address the special hair care needs of the Asian and African consumers. Therefore, the present study opens new chances for the development of lipid-related formulations for ethnic hair that can protection.

\section{REFERENCES}

1. Syed, A., Kuhajda, A., Ayoub, H., Ahmad, K., Frank, E.M. African-American hair: its physical properties and difference relative to Caucasian hair. Cosmet Toil. 110, 39-48 (1995).

2. Franbourg, A., Hallegot, P., Baltenneck, F., Toutain, C., and Leroy, F. Current research on ethnic hair. J. Am. Acad Dermatol, S115-S9 (2003).

3. Wolfram, L.J. Human hair: A unique physicochemical composite. J. Am. Acad. Dermatol. 48, S106-S114 (2003).

4. Ji J.H., Park, T.S., Lee, H.J., Kim, Y.D., Pi, L.Q., Jin, X.H., Lee W.S. The Ethnic Differences of the Damaged of Hair and Integral Hair Lipid after Ultra Violet Radiation. Ann. Dermatol 25, 54-60 (2013).

5. Cruz, C.F., Fernandes, M.M., Gomes, A.C., Coderch, L., Martí, M., Méndez, S., Gales, I., Azoia, N.G., Shimanovich, U., and Cavaco-Paulo, A. Keratins and lipids in ethnic hair. Int. J. Cosmet. Sci. 35, 244-249 (2013).

6. Kreplak, L., Briki, F., Duvault, Y., Doucet, J., Merigoux, C., Leroy, F., Lévêque, J.L., Miller, L., Carr, G.L., Williams, G.P., Dumas, P. Profiling lipids across Caucasian and Afro-American hair transverse cuts, using syncroton infrared microspectrometry. Int. J. Cosm. Sci. 23, 369-374 (2001).

7. Nicolaides, N., Rothman, S. Studies on the chemicak composition of human hair fat. II The overall composition with regards to age, sex and race. J. Invest. Dermat. 21, 9-12 (1953).

8. Wertz, P. W. \& Downing, D.T. Integral lipids of human hair. Lipids. 23, 878-881 (1988).

9. Jones, L.N. and River, D.E., 18-Methyleicosanoic acid in the structure and formation of mammalian hair fibres. Micron. 28, 469-485 (1997).

10. Coderch, L., S.Méndez, S., Barba, C., Pons, R., Martí, M., Parra, J.L. Lamellar rearrangement of internal lipids from human hair. Chem Phys Lipids. 155(1), 1-6 (2008).

11. Nishimura, K., Nishino, I., Inaoka, Y., Kitada, Y., Fukushima, M., Interrelatiomship between the hair lipids and the hair moisture, Nippon Koshohin Kagakkaishi 13, 134-139 (1989).

12. Barba, C., Martí, M., Carilla, J., Manich, A.M., Coderch, L. Moisture sorption/desorption of protein fibres. Thermochimica Acta. 552, 70-76 (2013).

13. Ohsawa, K., Watanabe, T., Matsukawa, R., Yoshimura, Y., Imaeda, K., The possible role of squalene and its peroxide of the sebum in the occurrence of sunburned and protection from the damage caused by U.V. irradiation. J. Toxico. Sci. 9, 151-159 (1984).

14. McMullen, R.L., Laura, D., Chen, S., Koelmel, D., Zhang, G., Gillece, T. Determination of physicochemical properties of delipidized hair. J. Cosmet. Sci. 64, 355-370 (2013).

15. Coderch, L., Oliver, M.A., Martínez, V., Manich, A.M., Rubio, L., Martí, M. Exogenous and endogenous lipids of human hair. Skin Res. Technol., 23, 479-485 (2017).

16. Martí, M., Barba, C., Manich, A.M., Rubio, L., Alonso, C. and Coderch, L., The influence of hair lipids in ethnic hair properties. Int. J. Cosmet. Sci. 38, 77-84 (2016). 
17. Molina, R., Comellas, F., Julià, M.R., Erra, P. Chemical modifications on human hair studied by means of contact angle determination. J. of Colloid and Interface Sci., 237, 40-46 (2001).

18. Schulze zur Wiesche, E., Körner, A., Scäfer, K. and Wortmann, F.J. Prevention of hair surface aging. J. Cosmet. Sci, 62, 237-249 (2011).

19. Anderson, R.B., Hall, W.K., Modifications of the Brunauer, Emmett and Teller Equation II1, J. Am. Chem. Soc. 70, 1727-1734 (1948).

20. Vickerstaff T. The physical chemistry of dyeing. London: Oliver and Boyd; 1954

21. Shaw, D.A. Hair lipid and surfactants. Extraction of lipid by surfactants and lack of shampooing on the rate of refatting of hair. Int. J. Cosmet. Sci. 1, 317-328 (1979).

22. Masukawa, Y., Tsujimura, H., Imokawa, G., A systematic method for the sensitive and specific determination of hair lipids in combination with chromatography. J. Chromatogr B Analyt Technol Biomed Life Sci. 823(2), 131-42 (2005).

23. Leeder, J.D., Rippon, J.A. Some observations on the dyeing of wool from aqueous formic acid. J. Soc. Dyers and Col. 99, 64-65 (1983).

24. Barba, C., Martí, M., Manich, A.M., Carilla, J., Parra, J.L., Coderch, L. Water absorption/desorption of human hair and nails. Thermochimica Acta. 503-504, 33-39 (2010).

25. Barba, C., Martí, M., Semenzato, A., Baratto, G., Manich, A.M., Coderch, L. Effect of lipid modification on stratum corneum permeability. J. Therm. Anal. Calorim. 120, 297-305 (2015). 
Figure 1. Quantification of external hair lipids in ethnic hairs using TLC/FID (percentage over the total weight of dry hair fiber (o.w.f)). Apolar lipids (Ap lip), free fatty acids (FFA), sterols (St) and polar lipids (P.lip). $(* p>0.05)$ $233 \times 131 \mathrm{~mm}(72 \times 72 \mathrm{DPI})$ 

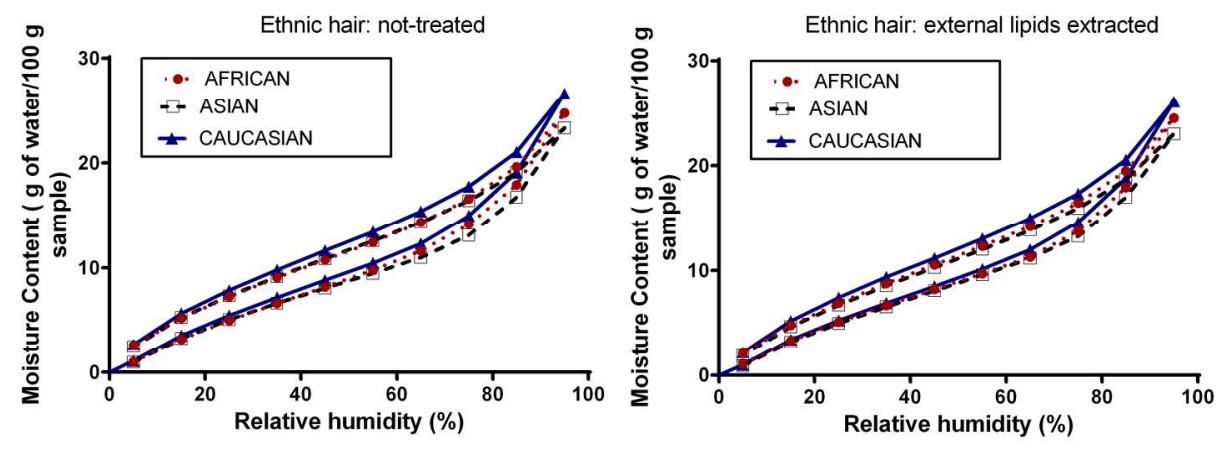

Figure 2. Water sorption isotherms of native ethnic hairs (A) and external lipid-extracted ethnic hairs (B) $560 \times 214 \mathrm{~mm}(100 \times 100 \mathrm{DPI})$ 
Table I. GAB model and parameters used to fit the experimental sorption data.

\begin{tabular}{|c|l|}
\hline Model & \multicolumn{1}{c|}{ Mathematical equation } \\
\hline $\mathrm{GAB}$ & $\mathrm{W}=\mathrm{Wm} \mathrm{Cg} \mathrm{K} \mathrm{w}_{\mathrm{w}} /\left[\left(1-\mathrm{Ka}_{\mathrm{w}}+\mathrm{Cg} \mathrm{K} \mathrm{a}_{\mathrm{w}}\right)\right]$ \\
\hline Parameter & \multicolumn{1}{c|}{ Definition } \\
\hline $\mathbf{a}_{\mathbf{w}}$ & Water activity expressed as vapor relative pressure $\mathrm{p} / \mathrm{p} 0$, where $\mathrm{p} 0$ is the saturated vapor. \\
\hline $\mathbf{W}$ & Equilibrium moisture content at aw in $\mathrm{g}$ sorbed/100 $\mathrm{g}$ of sorbent on a dry basis. \\
\hline $\mathbf{W m}$ & Monolayer moisture content in $\mathrm{g}$ sorbed/100 $\mathrm{g}$ of sorbent on a dry basis. \\
\hline $\mathbf{C g}$ & $\begin{array}{l}\text { Energy constant related to the difference between the free enthalpy of the water molecules } \\
\text { in the pure liquid state and in the monolayer, which is proportional to the rate between both } \\
\text { the attachment and the escape rate constants of the primary sites. }\end{array}$ \\
\hline $\mathbf{K}$ & $\begin{array}{l}\text { Ratio between the standard vapor pressure of the liquid and the vapor pressure of the } \\
\text { sorbate in the secondary (upper) layers, which is proportional to the rate between the } \\
\text { attachment rate constant and the escape for all higher layers. }\end{array}$ \\
\hline
\end{tabular}


Table II. Total amount of lipids obtained by extraction with tert-butanol /hexane (external lipids) and lipid analysis of the external extracted lipids from the different ethnic hairs

\begin{tabular}{|l|c|c|c|}
\hline Type of Hair & African & Asian & Caucasian \\
\hline TOTAL External lipids (\% o.f.w.) & $3.75 \pm 0.01$ & $0.48 \pm 0.16$ & $0.89 \pm 0.17$ \\
\hline APOL. L (\% o.t.a) & $51,1 \pm 6.2$ & $16,3 \pm 2.8$ & $24,8 \pm 12.6$ \\
\hline FFA (\% o.t.a) & $34,6 \pm 9.7$ & $60,7 \pm 3.9$ & $53,0 \pm 8.8$ \\
\hline ST. (\% o.t.a) & $10,2 \pm 8.4$ & $4,3 \pm 2.9$ & $8,1 \pm 5.1$ \\
\hline POL. L. (\% o.t.a) & $4,1 \pm 1.7$ & $18,7 \pm 5.4$ & $14,1 \pm 7.2$ \\
\hline
\end{tabular}

(o.w.f.: over weight of fiber; \% o.t.a.: over total analyzed) 
Table III. Melting point, DSC and TGA of external extracted lipids from the different ethnic hairs

\begin{tabular}{|c|c|c|c|c|}
\hline \multirow{2}{*}{} & \multicolumn{3}{|c|}{ EXTERNAL LIPIDS } \\
\cline { 3 - 5 } Melting point & \multirow{2}{*}{$\mathrm{C}$} & African & Asian & Caucasian \\
\hline DSC & $68.8 \pm 1.8$ & $55.0 \pm 3.5$ & $77.5 \pm 3.5$ \\
\hline \multirow{2}{*}{$\begin{array}{c}{ }^{\circ} \mathrm{C} ; \mathrm{Jg}^{-1} \\
\text { (phase transitions) }\end{array}$} & & $14.7 ;-17.7$ & $20.1 ;-32.0$ & $10.5 ;-24.7$ \\
\hline \multirow{2}{*}{$\begin{array}{c}\text { TGA } \\
\text { (degradation steps) }\end{array}$} & \multirow{2}{*}{${ }^{\circ} \mathrm{C} ; \%$} & $38.1 ;-6.6$ & $34.0 ;-9.8$ & $30.3 ;-11.5$ \\
\cline { 3 - 5 } & & $246.9 ; 43.3$ & $244.1 ; 61.8$ & $54.9 ;-6.4$ \\
\cline { 3 - 5 } & $332.9 ; 53.9$ & $360.4 ; 31.9$ & $237.1 ; 43.7$ \\
\hline
\end{tabular}


Table IV. Humidity, contact angle and radius evaluation of African, Asian and Caucasian fibers before and after lipid extraction. (H: humidity; Contact Angle (dynamic); $\varphi$ : Contact Angle (pseudo-static))

\begin{tabular}{|c|c|c|c|c|c|c|}
\hline \multirow{2}{*}{} & \multicolumn{2}{|c|}{ African } & \multicolumn{2}{c|}{ Asian } & \multicolumn{2}{c|}{ Caucasian } \\
\cline { 2 - 7 } & NT & Lip. Ext & NT & Lip. Ext & NT & Lip. Ext \\
\hline $\mathrm{H}(\%)$ & $11.05 \pm 1.36$ & $10.31 \pm 0.87$ & $10,75 \pm 0.96$ & $10.38 \pm 1.08$ & $11,65^{\star} \pm 0.92$ & $10.09^{*} \pm 1.2$ \\
\hline$\theta\left({ }^{\circ}\right)$ & $91.3^{\S} \pm 7.2$ & $82.8 \pm 37.0$ & $87.2^{\S} \pm 6.7$ & $86.5 \pm 17.5$ & $91.7^{\S} \pm 11.0$ & $82.5 \pm 17.6$ \\
\hline$\varphi\left({ }^{\circ}\right)$ & $118,8^{\S} \pm 10,0$ & $114,7 \pm 14,6$ & $100,1^{\S} \pm 18,3$ & $97,4 \pm 16,3$ & $112,3^{\S} \pm 17,9$ & $102,1 \pm 10,6$ \\
\hline
\end{tabular}

t-student $p<0.5 \quad$ *Evaluated between extracted and non-extracted fibers

$\S$ Evaluated between non-extracted fibers 
Table V. F: Fineness, IM: Initial modulus, D: Deformation (at break) and T: Breaking tenacity of the different non-treated and extracted hairs. The results are given as the mean values and confidence interval at $\pm 95 \%$.

\begin{tabular}{|c|c|c|c|c|c|c|}
\hline \multirow{2}{*}{} & \multicolumn{2}{|c|}{ African } & \multicolumn{2}{c|}{ Asian } & \multicolumn{2}{c|}{ Caucasian } \\
\cline { 2 - 6 } & NT & Lip. Ext & NT & Lip. Ext & NT & Lip. Ext \\
\hline $\begin{array}{c}\mathbf{F} \\
\text { tex (mg/m) }\end{array}$ & $6,82 \pm 0,37$ & $7,07 \pm 0,37$ & $6,28 \pm 0,36$ & $6,84 \pm 0,36$ & $5,82 \pm 0,36$ & $5,02 \pm 0,36 \downarrow$ \\
\hline $\begin{array}{c}\text { IM } \\
\mathbf{~ N / t e x}\end{array}$ & $3,257 \pm 0,231$ & $3,504 \pm 0,228$ & $4,298 \pm 0,228$ & $4,466 \pm 0,228$ & $4,155 \pm 0,228$ & $4,561 \pm 0,228$ \\
\hline $\begin{array}{c}\mathbf{D} \\
\%\end{array}$ & $35,83 \pm 0,93$ & $34,82 \pm 0,92$ & $39,09 \pm 0,92$ & $37,01 \pm 0,92 \downarrow$ & $39,35 \pm 0,92$ & $39,31 \pm 0,92$ \\
\hline $\begin{array}{c}\mathbf{T} \\
\mathbf{c N} / \mathrm{tex}\end{array}$ & $14,00 \pm 1,03$ & $13,54 \pm 1,02$ & $14,05 \pm 1,03$ & $14,20 \pm 1,03$ & $15,79 \pm 1,03$ & $18,04 \pm 1,05 \uparrow$ \\
\hline
\end{tabular}

Remarks: $\uparrow$ Significant increase or $\downarrow$ significant decrease at $1 \%$ level after lipid extraction 
Table VI. Maximum moisture regain, GAB monolayer capacity $(\mathrm{Wm})$, GAB energy constant $(\mathrm{Cg})$ constant $(\mathrm{K})$, GAB determination coefficient $\left(R^{2}\right)$, total time to reach equilibrium $\left(t_{T}\right)$ and apparent diffusion coefficient $\left(D_{A}\right)$ for native and external extracted ethnic hair fibers.

\begin{tabular}{|c|c|c|c|c|c|c|}
\hline \multirow{2}{*}{ Fiber Evaluation } & \multicolumn{2}{|c|}{ African } & \multicolumn{2}{c|}{ Asian } & \multicolumn{2}{c|}{ Caucasian } \\
\cline { 2 - 7 } & NT & Lip. Ext & NT & Lip. Ext & NT & Lip. Ext \\
\hline Regain at 95\% HR (\%) & 24.60 & 23.92 & 23.23 & 23.28 & 26.25 & 25.61 \\
\hline $\mathbf{W m}(\%)$ & 0.0746 & 0.0704 & 0.0743 & 0.0718 & 0.0772 & 0.0732 \\
\hline $\mathbf{C g}$ & 5.264 & 5.956 & 5.083 & 5.821 & 5.451 & 5.848 \\
\hline $\mathbf{K}$ & 0.7299 & 0.7403 & 0.7071 & 0.7251 & 0.7367 & 0.7481 \\
\hline $\mathbf{R}^{2}$ & 0.9987 & 0.9975 & 0.9972 & 0.9972 & 0.9980 & 0.9977 \\
\hline $\mathbf{t}_{\mathbf{T}}(\mathrm{min})$ & 3204 & 3116 & 3243 & 3277 & 3318 & 3328 \\
\hline $\mathbf{D}_{\mathbf{A}}\left(\mathrm{min}^{-1} \times 10^{-3}\right)$ & 21.2 & 22.5 & 21.1 & 20.6 & 20.5 & 20.3 \\
\hline $\mathbf{D}_{\text {AD }}\left(\mathrm{min}^{-1} \times 10^{-3}\right)$ & 27.1 & 27.7 & 26.4 & 25.3 & 25.0 & 25.0 \\
\hline
\end{tabular}


Table VII. ATR/FTIR of $\mathrm{CH}_{2}$ symmetric stretching frequencies (related to lipid order) and $\mathrm{CH}_{2}$ asymmetric stretching integration normalized with Amide I (related to lipid amount), of native and external extracted ethnic hair fibers in the cuticle and in the cortex.

\begin{tabular}{|c|c|c|c|c|c|c|}
\hline $\begin{array}{c}\text { Fibres } \\
\text { Evaluation }\end{array}$ & \multicolumn{2}{|c|}{ African } & \multicolumn{2}{c|}{ Asian } & \multicolumn{2}{c|}{ Caucasian } \\
\cline { 2 - 7 } & NT & Lip. Ext & NT & Lip. Ext & NT & Lip. Ext \\
\hline $\begin{array}{c}\text { Lip. Order CUT } \\
\mathrm{CH}_{2} \text { St.Sym }\left(\mathrm{cm}^{-1}\right)\end{array}$ & $2852.3 \pm 0.8$ & $2852.8 \pm 0.8$ & $2851.9 \pm 0.8$ & $2851.5 \pm 0.7$ & $2852.5 \pm 0.6$ & $2851.1 \pm 0.7$ \\
\hline $\begin{array}{c}\text { Lip. Amount CUT } \\
\mathrm{CH}_{2} \text { St. Asym/Amide I }\end{array}$ & $1.029 \pm 0.038$ & $0.907 \pm 0.022$ & $0.92 \pm 0.027$ & $0.929 \pm 0.023$ & $0.967 \pm 0.033$ & $0.960 \pm 0.063$ \\
\hline $\begin{array}{c}\text { Lip. Order CORTEX } \\
\left.\mathrm{ACH}_{2} \text { St.Sym (cm }{ }^{-1}\right)\end{array}$ & $2852.9 \pm 0.9$ & $2852.9 \pm 1.2$ & $2852.1 \pm 1.4$ & $2852.6 \pm 0.65$ & $2851.7 \pm 0.6$ & $2852.0 \pm 0.39$ \\
\hline $\begin{array}{c}\text { Lip. Amount CORTEX } \\
\mathrm{CH}_{2} \text { St. Asym/Amide I }\end{array}$ & $1.070 \pm 0.054$ & $0.886 \pm 0.027$ & $0.914 \pm 0.028$ & $0.903 \pm 0.029$ & $0.939 \pm 0.030$ & $0.887 \pm 0.053$ \\
\hline
\end{tabular}

${ }^{*} \mathrm{p}>0.05$ respect NT 